\title{
$h$-Adaptive Finite Element Solution of High Rayleigh Number Thermally Driven Cavity Problem
}

\author{
David A. Mayne, Asif S. Usmani and Martin Crapper \\ School of Civil and Environmental Engineering, University of Edinburgh
}

\section{Abstract}

An $h$-adaptive finite element code for solving coupled Navier-Stokes and energy equations is used to solve the thermally driven cavity problem. The buoyancy forces are represented using the Boussinesq approximation. This problem is characterised by very thin boundary layers at high values of Rayleigh number $\left(>10^{6}\right)$. However steady state solutions are achievable with adequate discretisation. This is where the auto-adaptive finite element method provides a powerful means of achieving optimal solutions without having to pre-define a mesh, which may be either inadequate or too expensive. Steady state and transient results are given for six different Rayleigh numbers in the range $10^{3}$ to $10^{8}$ for a Prandtl number of 0.71 . The use of $h$-adaptivity, based on a-posteriori error estimation is found to ensure a very accurate problem solution at a reasonable computational cost.

\section{Introduction}

Thermally driven cavity flow has been used to validate computer fluid flow models for the last two decades, it is also an important flow problem in its own right. Simulating a large horizontal temperature difference across a vertical cavity has applications in nuclear reactor insulation, double glazing, predicting fire spread in buildings and dispersion of heat in estuaries. The thermal cavity problem has been extensively studied and solved for Prandtl number of 0.71 (corresponding to an air filled cavity ) over a range of Rayleigh numbers in a steady state [1-4], and transient manner [5-7]. Several researchers have solved this problem using $\operatorname{Pr}=1.0$ [8-10], however Patterson and Imberger [11] determined that the steady state result is independent of the Prandtl number, but the transient behaviour and hence the approach to steady state, is not. De Vahl Davis [1] invited researchers to submit solutions of the thermal cavity problem using $\operatorname{Pr}=0.71$. A complete set of results is available for comparison of flow data at Rayleigh numbers from $10^{3}$ to $10^{6}$. De Vahl Davis concluded that, for a given problem and solution technique, mesh density controlled the accuracy of the results. 
He also concluded that although there were accurate contributions from both the FEM and FDM the former was by and large the better, giving better results at higher Rayleigh numbers. De Vahl Davis also recommends that further work be conducted on the selective refinement of the mesh in the region of the boundary layers, stressing that this would be important for high Rayleigh numbers.

Finally he reports that Upson et al, who produced one of the best solutions, used the finite element method and had taken care to provide a high density of grid points in the wall and corner regions of the cavity.

Solutions have been obtained for Rayleigh numbers of $10^{7}$ and $10^{8}$ by a number of authors, for instance [3,5,12-14], but the volume of published results is considerably less than for the lower Rayleigh number cases. Solutions for Rayleigh numbers $10^{3}$ to $10^{8}$ will be presented in this paper and compared with the existing results.

\section{Governing equations}

The governing equations have been written for a constant density, incompressible Newtonian fluid using the Boussinesq approximation to model buoyancy.

Continuity

$$
\nabla \cdot \mathrm{v}=0
$$

where $\mathbf{v}$ represents the velocity.

Navier-Stokes

$$
\rho\left(\frac{\partial \mathbf{v}}{\partial t}+\mathbf{v} \cdot \nabla \mathbf{v}\right)+\nabla P=\nabla \cdot \mu\left[\nabla \mathbf{v}+(\nabla \mathbf{v})^{T}\right]-\rho \mathbf{g} \beta\left(T-T_{r}\right)
$$

subject to boundary conditions:

$$
\begin{gathered}
\mathbf{F}=P \mathbf{n}-\mu\left[\nabla \mathbf{v}+(\nabla \mathbf{v})^{T}\right] \cdot \mathbf{n} \\
\mathbf{v}=\overline{\mathbf{v}}(x, y, t)
\end{gathered}
$$

and initial conditions:

$$
\mathbf{v}(t=0)=\mathbf{v}_{\mathbf{o}} \quad \text { with } \quad \nabla \cdot \mathbf{v}_{\mathbf{o}}=0
$$


$\mu$ is the dynamic viscosity, $\mathrm{g}$ is the acceleration due to gravity, $\beta$ is the volumetric coefficient of thermal expansion, $T$ is the temperature, $T_{r}$ is a reference temperature, $\mathbf{F}$ represents the applied tractions on the boundary and $n$ is the unit normal vector.

\section{Energy}

$$
\frac{\partial T}{\partial t}+\mathbf{v} \cdot \nabla T=\nabla \cdot \kappa \nabla T
$$

subject to boundary conditions:

$$
\begin{aligned}
& \mathbf{n} \cdot(\kappa \nabla T)=q \\
& T=\bar{T}(x, y, t)
\end{aligned}
$$

and initial conditions:

$$
T(t=0)=T_{o}
$$

where $q$ is a specified normal heat flux and $\kappa$ is the thermal diffusivity given by,

$$
\kappa=\frac{k}{\rho C_{p}}
$$

where, $k$ is the thermal conductivity, $\rho$ is the fluid density and $C_{p}$ is the specific heat capacity.

\section{$2.1 \quad$ Finite Element Formulation}

The program is based on the Galerkin Finite Element Method (GFEM), solving for the primitive variables: U-velocity, $\mathrm{V}$-velocity and T-temperature at all nodes in the mesh and P-pressure at a reduced level of interpolation to avoid spurious pressure modes, using a mixed formulation for the Navier-Stokes equations. The Navier-Stokes and energy equations were coupled by the Boussinesq approximation for buoyancy. Notation used here is as used by Gresho et al, [8,15]. The Galerkin FEM discretisation produces a system of ODE's as follows:

Navier-Stokes

$$
\left[\begin{array}{ccc}
\mathbf{M}_{\mathbf{u}} & 0 & 0 \\
0 & 0 & 0 \\
0 & 0 & \mathbf{M}_{\mathbf{v}}
\end{array}\right]\left(\begin{array}{c}
\dot{\mathbf{u}} \\
\dot{\mathbf{P}} \\
\dot{\mathbf{v}}
\end{array}\right)+\left[\begin{array}{ccc}
\mathbf{K}_{\mathbf{u u}} & \mathrm{C}_{\mathbf{u}} & \mathbf{K}_{\mathbf{u v}} \\
\mathbf{C}_{\mathbf{u}}^{\mathbf{T}} & 0 & \mathbf{C}_{\mathbf{v}}^{\mathbf{T}} \\
\mathbf{K}_{\mathbf{v u}} & \mathbf{C}_{\mathbf{v}} & \mathbf{K}_{\mathbf{v v}}
\end{array}\right]\left(\begin{array}{c}
\mathbf{u} \\
\mathbf{P} \\
\mathbf{v}
\end{array}\right)=\left(\begin{array}{c}
\mathbf{F}_{\mathbf{u}} \\
0 \\
\mathbf{F}_{\mathbf{v}}
\end{array}\right)
$$


The first to third rows represent the $x$-momentum, continuity and $y$-momentum equation respectively. The right hand side vector $\mathbf{F}_{v}$ contains the coupling buoyancy term.

Energy

$$
\left[\mathbf{M}_{\mathbf{T}}\right](\dot{\mathbf{T}})+\left[\mathbf{K}_{\mathbf{T}}\right](\mathbf{T})=\left(\mathbf{F}_{\mathbf{T}}\right)
$$

Expansion of all terms can be found in Usmani et al [16]. The two systems of equations above are solved as a coupled system, with the $\mathbf{K}_{\mathbf{T}}$ term containing the velocities (obtained from solving the flow field) and the $\mathbf{F}_{\mathbf{v}}$ term containing the buoyancy forces (determined by the temperature field).

\subsection{Temporal discretisation}

Temporal discretisation of the time domain is achieved by applying the generalised midpoint rule, [17, 18].

$$
\left[\frac{\mathbf{M}_{\mathbf{n}+\alpha}}{\alpha \Delta t}+\mathbf{K}_{\mathbf{n}+\alpha}\right]\left(\theta_{n+1}\right)=\left[\frac{\mathbf{M}_{\mathbf{n}+\alpha}}{\alpha \Delta t}-\frac{(1-\alpha)}{\alpha} \mathbf{K}_{\mathbf{n}+\alpha}\right]\left(\theta_{n}\right)+\frac{\left(\mathbf{F}_{\mathbf{n}+\alpha}\right)}{\alpha}
$$

Variation of $\alpha$ leads to different members of this family of methods i.e.

$\alpha=0$-Forward Difference or Forward Euler.

$\alpha=\frac{1}{2}$-Midpoint rule or Crank Nicolson.

$\alpha=\frac{2}{3}$-Galerkin.

$\alpha=1$-Backward Difference or Backward Euler.

The Crank Nicolson, Galerkin and Backward Euler schemes are all unconditionally stable, however, of these methods the oscillation limit is lowest for $\alpha=\frac{1}{2}$. A larger time step size chosen for $R a \rightarrow 10^{3}$ to $10^{7}$ constrains the choice of $\alpha$ to $\frac{2}{3}$. The time step size for $R a=10^{8}$ is chosen to be small enough to avoid an oscillatory solution when using $\alpha=\frac{1}{2}$. The choice of unconditionally stable implicit methods is enforced by the use of $h-$ adaptivity as the smallest elements determine the stability of conditionally stable explicit methods, which makes them impractical for use in this context.

The formulations described above were implemented in the implicit transient FE code CADTRAS (Coupled Advective Diffusive TRAnSport model), which was used to solve the thermally driven cavity problem. The code incorporates an unstructured Delaunay triangulation based mesh generator [19], which allows automatic adaptive re-meshing to take place at each time step if necessitated by the a-posteriori error estimation algorithm. Six-node triangular elements are used for all the meshes. 


\section{Adaptivity}

The use of $h$-adaptivity enables the solution of this problem at high Rayleigh number without the necessity of designing a suitable mesh at first and going through a trial-and-error process. Adaptivity automatically produces an optimal mesh based on a user specified discretisation error thus saving computational time and focusing effort intelligently over successive time steps on areas of high scalar gradients (which for this problem coincide with the areas of high velocity gradients). There are five distinct steps to the iterative adaptive process used here :

1. Solution of the coupled system

2. Recovery of smoothed scalar gradients using the super-convergent patch recovery (SPR) method [20]

3. Error Estimation using the a-posteriori error calculated at all nodes in the mesh for the scalar field

4. Re-meshing based on the mesh sizes produced from the previous step

5. Transfer of all data to the new mesh

\section{Recovery}

The temperature field generated by the finite element method is most accurate at nodal points whereas the temperature gradients are most accurate at Gaussian integration points, known as the super-convergence phenomenon. Hinton and Campbell [21] showed that finite elements produce superior values of temperature gradient at node points after application of a smoothing procedure. Their method was based on a global smoothing scheme requiring the solution of a large system of equations. A more efficient and effective procedure was introduced by Zienkiewicz and Zhu [20], called super-convergent patch recovery (SPR). The smoothed nodal gradients are calculated from the Gauss points on a patch of elements surrounding a node, using a least squares interpolation, for each node in the mesh.

\section{Error Estimation}

The error estimator used was originally derived for heat conduction [22]. Mathematical justification of using such an estimator for the problem of this paper does not exist, however as the estimator used is based on the scalar flux, it has proven very effective in detecting regions of high scalar gradient, which in practice is sufficient for the purposes of this paper. The a-posteriori error is based upon an energy norm (see [22]),

$$
\|e\|^{2}=\int_{\Omega}(\nabla T)^{T} \kappa \nabla T d \Omega-\int_{\Omega}(\nabla \hat{T})^{T} \kappa \nabla \hat{T} d \Omega
$$


if we define,

$$
\begin{aligned}
& \|Q\|^{2}=\int_{\Omega}(\nabla T)^{T} \kappa \nabla T d \Omega \\
& \|\hat{Q}\|^{2}=\int_{\Omega}(\nabla \hat{T})^{T} \kappa \nabla \hat{T} d \Omega
\end{aligned}
$$

then Equation (12) can be rewritten as

$$
\|e\|^{2}=\|Q\|^{2}-\|\hat{Q}\|^{2}
$$

Such a definition allows a practical representation of the error norm in terms of a percentage error $\eta$,

$$
\eta=\frac{\|e\|}{\|Q\|} \times 100 \%
$$

\section{Re-meshing and mesh generation}

Specification of a permissible error $\bar{\eta}$ determines the level of refinement throughout the mesh, leading to a predicted reduction or increase in the element sizes so that the new mesh may possess an approximately equal distribution of error. The maximum permissible error for each element is calculated as,

$$
\|\hat{e}\|_{e}=\bar{\eta}\left(\frac{\|Q\|^{2}}{m}\right)^{\frac{1}{2}}
$$

where $m$ is the number of elements, $\bar{\eta}$ is the specified maximum percentage error. Dividing $\|\hat{e}\|_{\epsilon}$ by the calculated error in an element yields a parameter $\xi_{\epsilon}$ as follows,

$$
\xi_{e}=\frac{\|e\|_{e}}{\|\hat{e}\|_{e}}
$$

i.e. if $\xi_{e}>1$ the mesh must be refined in the vicinity of element $e$, conversely, if $\xi_{\epsilon}<1$ the mesh may be coarsened. The new element size is calculated using,

$$
\bar{h}_{e}=\frac{h_{e}}{\xi_{e}^{\frac{1}{p}}}
$$

where $h_{\epsilon}$ is the original element size and $p$ is the order of the element shape functions.

\section{Mesh data transfer}

Ensuring proper transfer of variables between meshes is crucial for conservation of quantities such as energy and momentum. A transfer strategy using local coordinates of nodal points and elemental shape functions has been used that maps the mesh data accurately. The local coordinates $(\xi-\eta)$ of each node in the adapted mesh are determined with respect to the elements of the previous mesh. Element shape functions are then used to interpolate the data onto the new mesh nodes. 


\section{The thermally driven cavity benchmark problem}

The problem involves modelling fluid flow in a two dimensional square cavity of typical dimension $L$ with the two vertical walls being maintained at a temperature difference of $\Delta T$ (see Figure 1). The top and bottom walls are insulated and the velocities at all boundaries set to zero. The fluid inside the cavity is initially at rest and at a temperature which is the mean of the temperatures on the vertical walls.

$$
\begin{array}{ll}
\mathrm{u}=0 & \mathrm{dT} / \mathrm{d} \mathbf{y}=\mathbf{0} \\
\mathrm{v}=\mathrm{0} &
\end{array}
$$

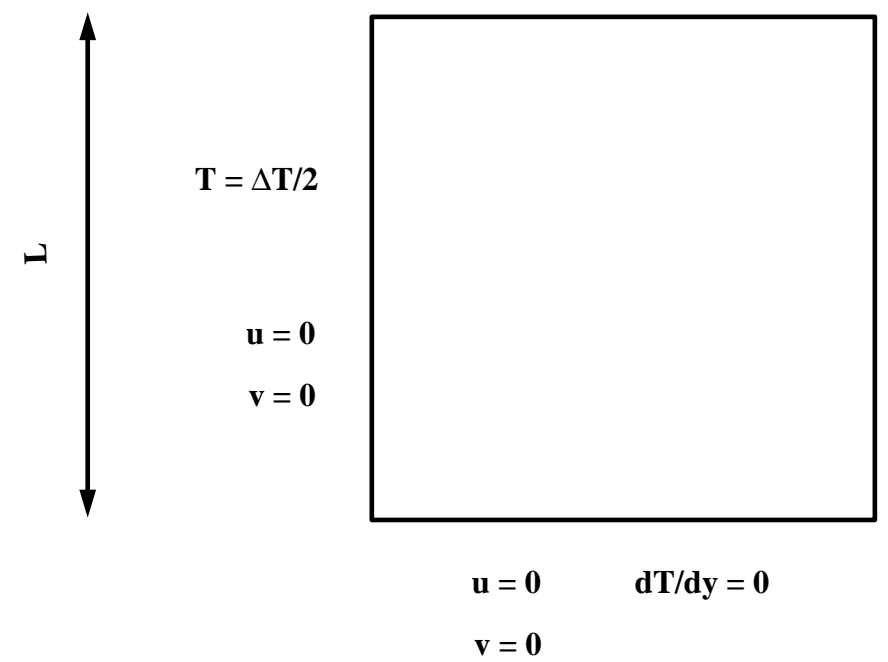

$\mathbf{T}=-\Delta \mathbf{T} / 2$

$\mathbf{u}=\mathbf{0}$

$\mathbf{v}=\mathbf{0}$

Figure 1: Boundary conditions for thermal cavity benchmark problem

The steady state flow and heat transfer in the thermal cavity is characterised by the Rayleigh number

$$
R a=g \beta \frac{\Delta T L^{3}}{\nu \kappa}
$$

The following non-dimensional groups are used in the analysis and presentation of the computational results:

Velocity

$$
\begin{aligned}
& u^{*}=\frac{u L}{\kappa} \\
& v^{*}=\frac{v L}{\kappa}
\end{aligned}
$$


Temperature

$$
T^{*}=\frac{T-T_{2}}{T_{1}-T_{2}}
$$

\section{Coordinates}

$$
\begin{aligned}
& x^{*}=\frac{x}{L} \\
& y^{*}=\frac{y}{L}
\end{aligned}
$$

Time

$$
t^{*}=\frac{\kappa t}{L^{2}}
$$

where ${ }^{*}$ indicates the the non-dimensional quantity. $T_{1}$ and $T_{2}$ are the fixed temperatures at the two side walls of the cavity.

The Nusselt number is calculated at each node in the domain using

$$
N u=u T-\frac{\partial T}{\partial x}
$$

where the temperature gradient is obtained by the gradient recovery process.

\section{Results}

The spacing between the isotherms at the sides of the cavity decreases with increasing Rayleigh number. The high level of mesh refinement in this area allows the thin boundary layer to be captured accurately, avoiding any oscillations that can be generated when modelling steep temperature gradients without adequate discretisation. A uniformly distributed mesh with the 'same' number of elements will be inadequate to model large changes in temperature at the boundary and will produce an oscillatory solution. At high Rayleigh numbers $\left(>10^{6}\right)$ a convergent solution may not be possible at all. Figure 2 shows the top half of the cavity for four different dimensionless times. It can be seen that the mesh adapts to follow the high temperature gradient front as it passes the departing corner. The refinement of the mesh around the side walls at $t=0.0001$ (Figure 2(a)) is due to a pre-adaptive loop where the mesh is refined based on the the initial conditions.

Figure 3 shows plots of velocity and temperature along the centre line of the cavity $(y=0)$ for each Rayleigh number at steady state. The figure clearly shows that mesh refinement 


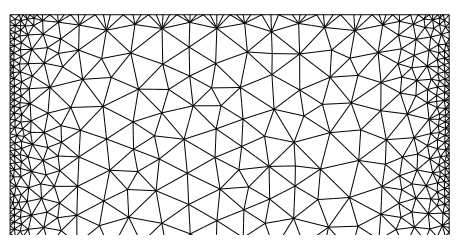

(a) $t^{*}=0.0001$, mesh

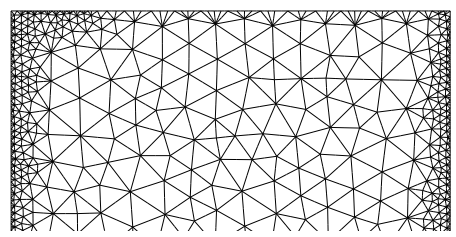

(d) $t^{*}=0.0005$, mesh

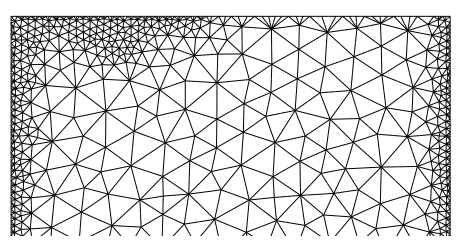

(g) $t^{*}=0.0008$, mesh

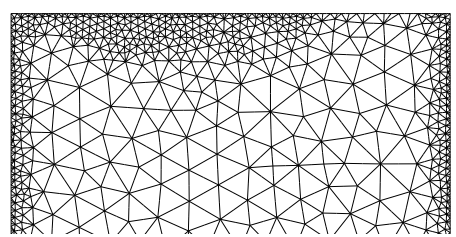

(j) $t^{*}=0.001$, mesh

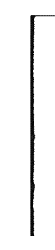

(b) $t^{*}=0.0001$, isotherms

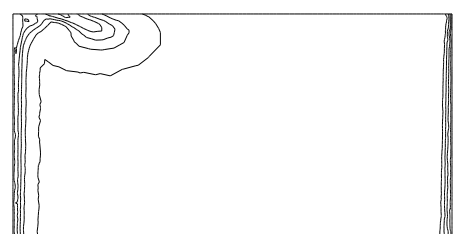

(e) $t^{*}=0.0005$, isotherms

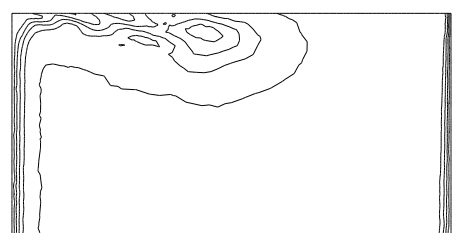

(h) $t^{*}=0.0008$, isotherms

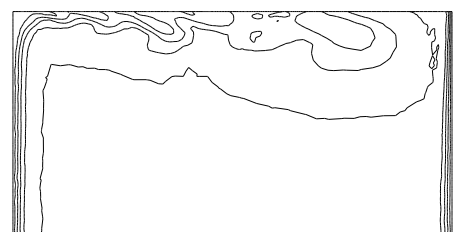

(k) $t^{*}=0.001$, isotherms

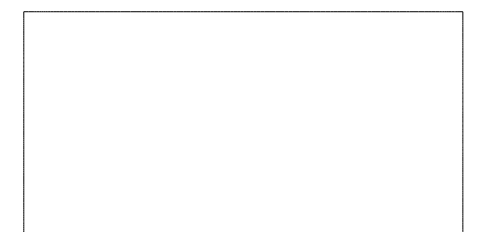

(c) $t^{*}=0.0001$, velocity vectors

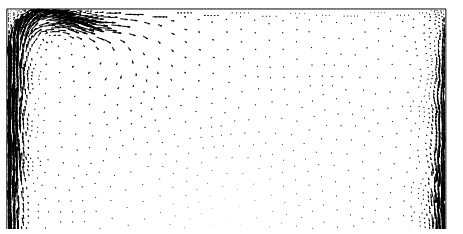

(f) $t^{*}=0.0005$, velocity vectors

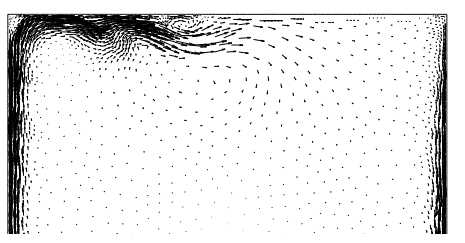

(i) $t^{*}=0.0008$, velocity vectors

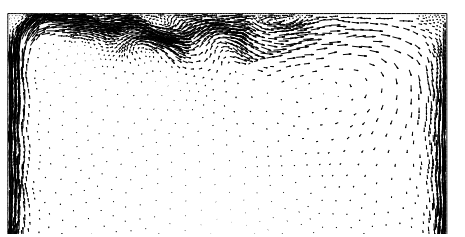

(1) $t^{*}=0.001$, velocity vectors

Figure 2: Mesh development for $\mathrm{Ra}=10^{8}$. 


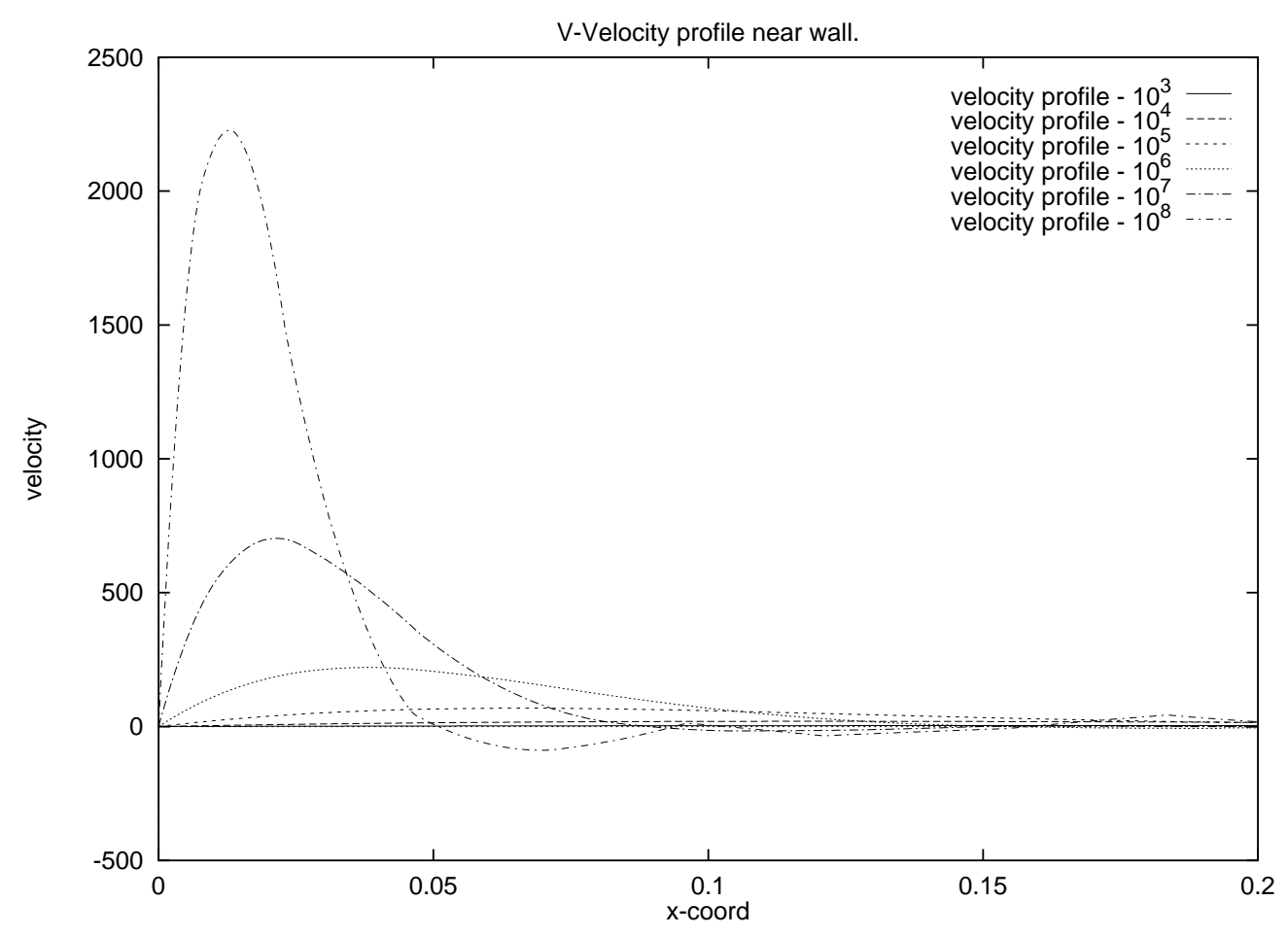

(a) Plot of velocity on $y=0.5$ near boundary

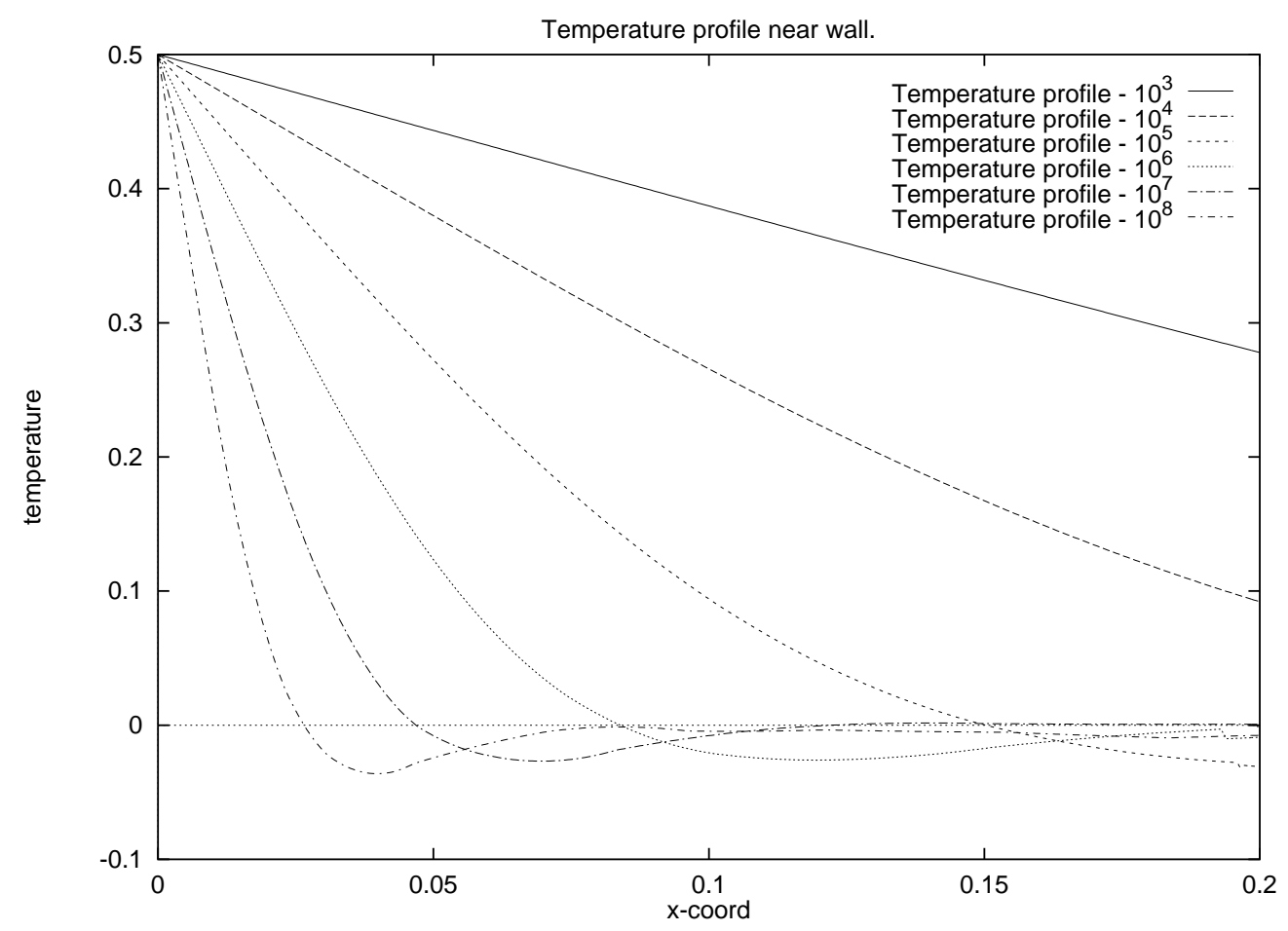

(b) Plot of temperature on $y=0.5$ near boundary

Figure 3: Velocity and temperature near the vertical boundary 


\begin{tabular}{|c|l|}
\hline Parameter & Description \\
\hline$U_{\max }$ & Maximum horizontal velocity on vertical mid plane of cavity \\
\hline$y$ & y-coordinate position of maximum horizontal velocity on vertical mid plane \\
\hline$V_{\max }$ & Maximum vertical velocity on horizontal mid plane of cavity \\
\hline$x$ & x-coordinate position of maximum vertical velocity on horizontal mid plane \\
\hline$N u_{0}$ & Average Nusselt number on the vertical boundary $x=0$ \\
\hline$N u_{\max }$ & Maximum Nusselt number on the vertical boundary $x=0$ \\
\hline$y$ & y-coordinate position of maximum Nusselt number on vertical boundary $x=0$ \\
\hline$N u_{\min }$ & Minimum Nusselt number on the vertical boundary $x=0$ \\
\hline$y$ & y-coordinate position of minimum Nusselt number on vertical boundary $x=0$ \\
\hline
\end{tabular}

Table 1: Measured parameters

based only upon the temperature gradients is appropriate for this problem as the steep velocity gradients occur in the same locations as the steep temperature gradients.

Figures 4 to 9 show the mesh, velocity vectors, pressure field and isotherms for each Rayleigh number at steady state.

Table 2 shows results obtained for the six Rayleigh numbers investigated. The parameters presented are described in table 1.

$R a \rightarrow 10^{3}$ to $10^{6}$

The results for all the values measured are very close to the accurate results obtained by De Vahl Davis [4], only deviating slightly at $10^{6}$. The error between De Vahl Davis' solution and the adaptive FEM results have been calculated and presented in table 3 , in the manner presented in reference [1].

$R a \rightarrow 10^{7}$ to $10^{8}$

Established benchmark solutions analogous to the lower Rayleigh number results do not exist for the higher Rayleigh number problems. However, several researchers have solved the thermally driven cavity problem for $10^{7}$ and above. Table 4 shows the comparison of the adaptive FEM results with others for the Rayleigh number of $10^{7}$. Again the adaptive FEM results compare well with other solutions both in the magnitude of velocity and heat transfer rates but also with the locations of the maximum and minimum values. A similar comparison is shown in Table 5 for Rayleigh number $10^{8}$, the same conclusions apply. 


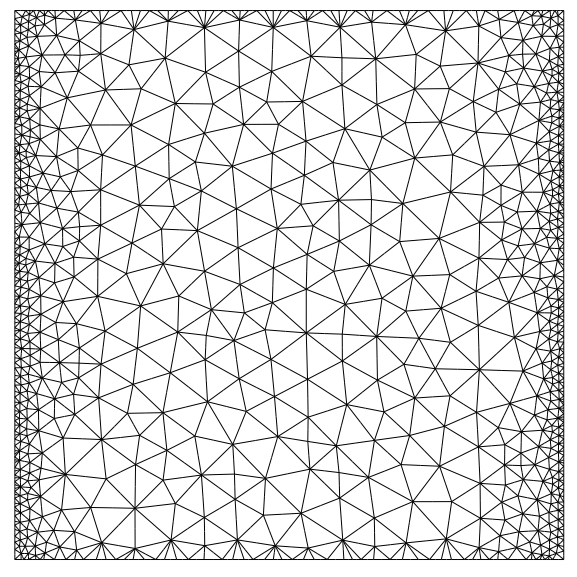

(a) Mesh

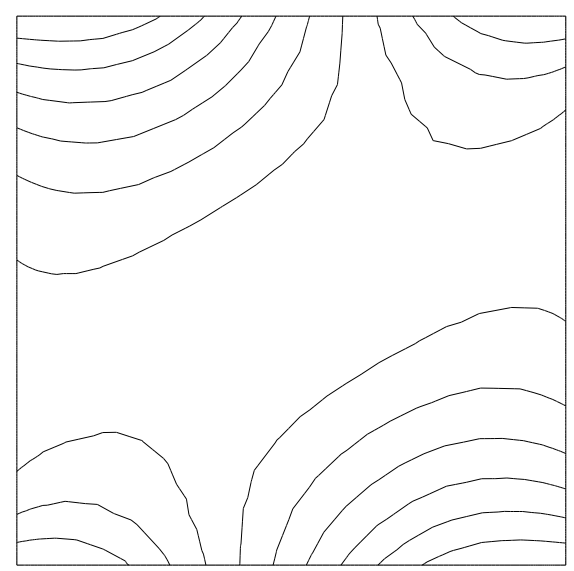

(c) Pressure

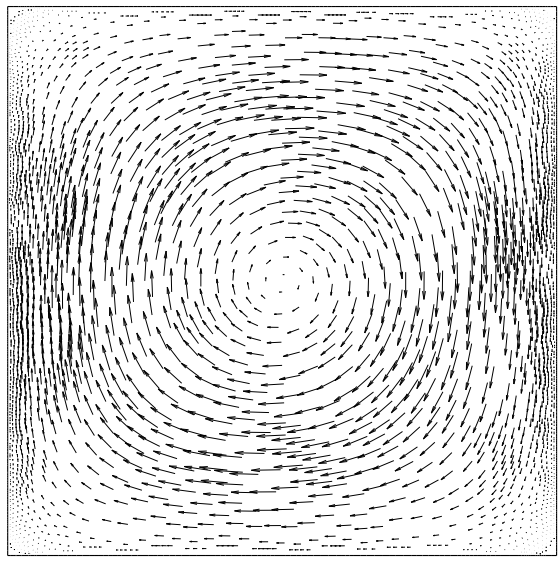

(b) Velocity Vectors

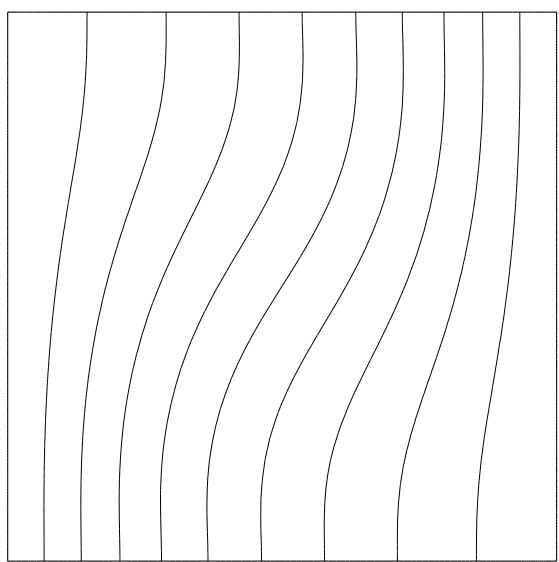

(d) Temperature

Figure 4: Above figures show the mesh, velocity vector plot, temperature contours and pressure contours for a Rayleigh number of $10^{3}$

\begin{tabular}{|c|c|c|c|c|c|c|}
\hline & $10^{3}$ & $10^{4}$ & $10^{5}$ & $10^{6}$ & $10^{7}$ & $10^{8}$ \\
\hline \hline$U_{\max }$ & 3.6493 & 16.1798 & 34.7741 & 64.6912 & 145.2666 & 283.0689 \\
$y$ & 0.8125 & 0.8235 & 0.8535 & 0.8460 & 0.8845 & 0.9455 \\
\hline$V_{\max }$ & 3.6962 & 19.6177 & 68.6920 & 220.8331 & 703.2526 & 2223.4424 \\
$x$ & 0.1790 & 0.1195 & 0.0665 & 0.0380 & 0.0215 & 0.0130 \\
\hline$N u_{0}$ & 1.1149 & 2.2593 & 4.4832 & 8.8811 & 16.3869 & 29.6256 \\
\hline$N u_{\max }$ & 1.5062 & 3.5305 & 7.7084 & 17.5308 & 41.0247 & 91.2095 \\
$y$ & 0.08956 & 0.1426 & 0.08353 & 0.03768 & 0.03899 & 0.0670 \\
\hline$N u_{\min }$ & 0.6913 & 0.5850 & 0.7282 & 0.9845 & 1.3799 & 2.0440 \\
$y$ & 1.0 & 1.0 & 1.0 & 1.0 & 1.0 & 1.0 \\
\hline
\end{tabular}

Table 2: The benchmark solution 


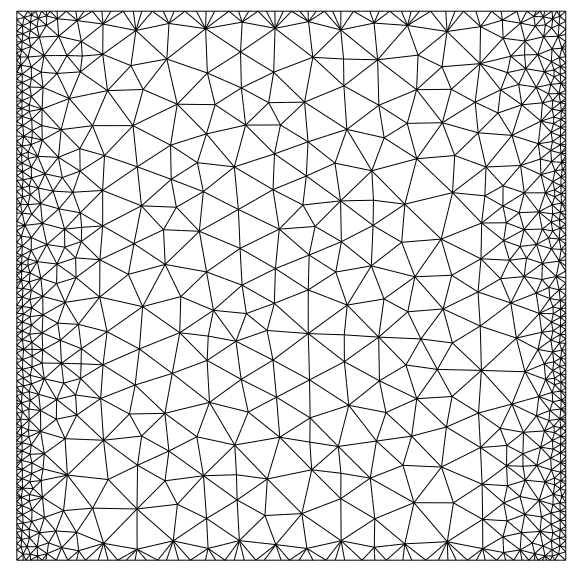

(a) Mesh

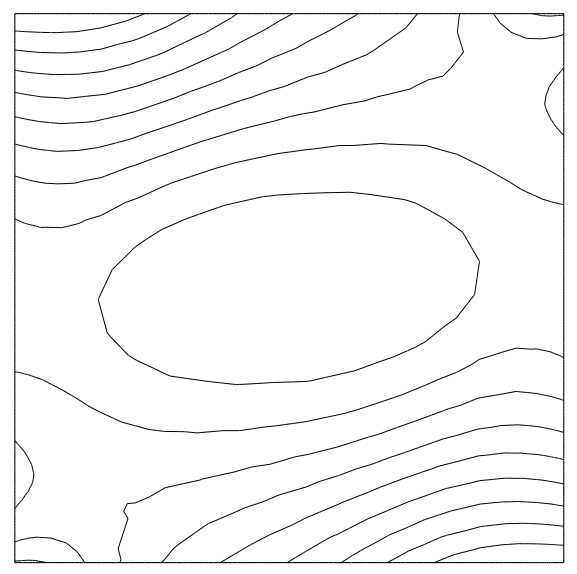

(c) Pressure

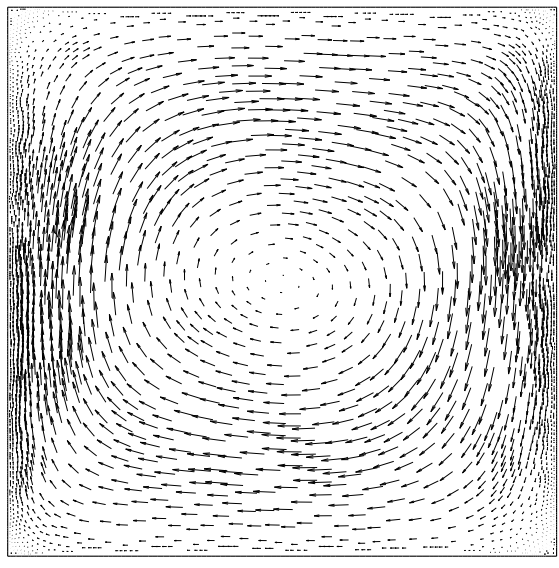

(b) Velocity Vectors

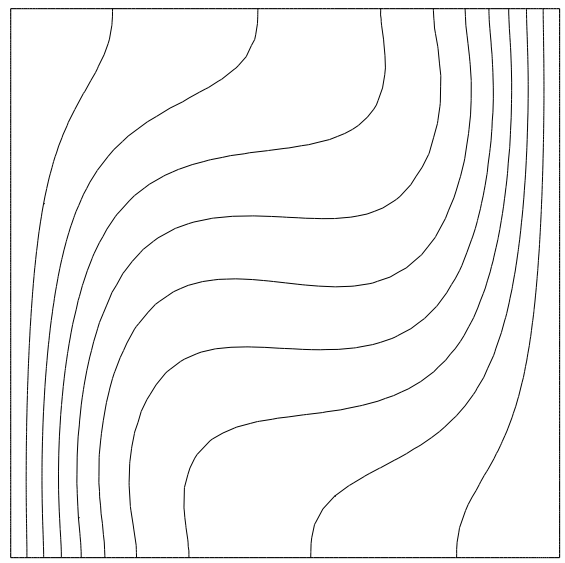

(d) Temperature

Figure 5: Above figures show the mesh, velocity vector plot, temperature contours and pressure contours for a Rayleigh number of $10^{4}$

\begin{tabular}{|c|c|c|c|c|}
\hline & $10^{3}$ & $10^{4}$ & $10^{5}$ & $10^{6}$ \\
\hline \hline$U_{\max }$ & 0.0 & 0.0 & -0.1 & -0.1 \\
\hline$V_{\max }$ & 0.0 & 0.0 & -0.1 & -0.7 \\
\hline$N u_{0}$ & 0.2 & -1.0 & 0.6 & -0.7 \\
\hline$N u_{\max }$ & -0.1 & -0.1 & 0.1 & 2.2 \\
\hline$N u_{\min }$ & 0.1 & 0.2 & 0.1 & 0.5 \\
\hline
\end{tabular}

Table 3: Solution error (\%) 


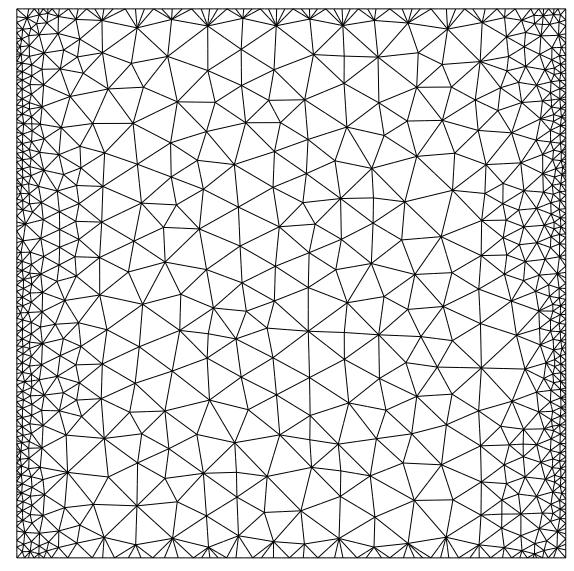

(a) Mesh

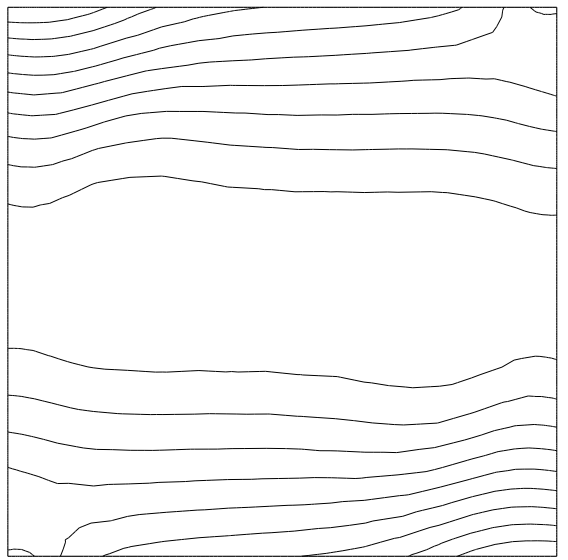

(c) Pressure

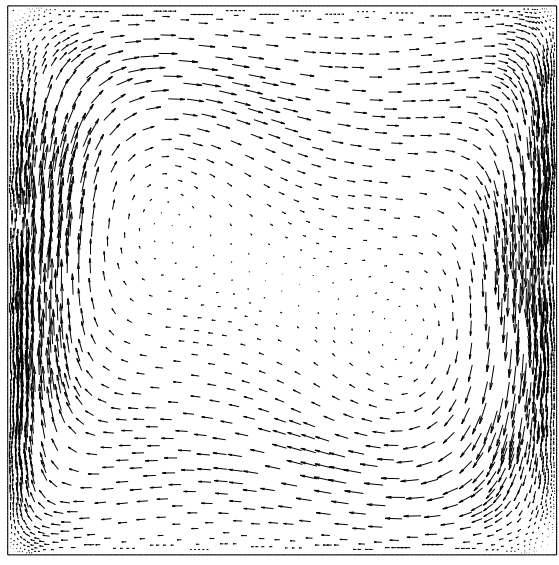

(b) Velocity Vectors

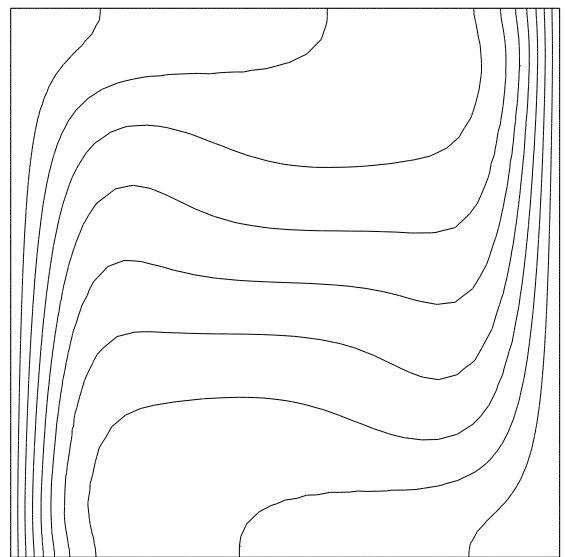

(d) Temperature

Figure 6: Above figures show the mesh, velocity vector plot, temperature contours and pressure contours for a Rayleigh number of $10^{5}$

\begin{tabular}{|c|c|c|c|c|c|}
\hline & Present work & Haldenwang [12,13] & Le Quere [5] & Chenoweth [6] & Le Quere [14] \\
\hline \hline$U_{\max }$ & 145.26 & 381.6 & 148.6 & 146 & 148.8 \\
$y$ & 0.8845 & & 0.879 & 0.881 & 0.879 \\
\hline$V_{\max }$ & 703.2526 & 700.4 & 699.1 & 699 & 699.3 \\
$x$ & 0.0215 & & 0.021 & 0.0213 & 0.0213 \\
\hline$N u_{0}$ & 16.3869 & 16.53 & 16.523 & 16.82 & 16.51 \\
\hline$N u_{\max }$ & 41.0247 & & 39.39 & & 39.37 \\
$y$ & 0.03899 & & 0.018 & & 0.0180 \\
\hline$N u_{\min }$ & 1.3799 & & 1.36635 & & 1.367 \\
$y$ & 1.0 & & 1.0 & & 1.0 \\
\hline
\end{tabular}

Table 4: The benchmark solution for $R a=10^{7}$ 


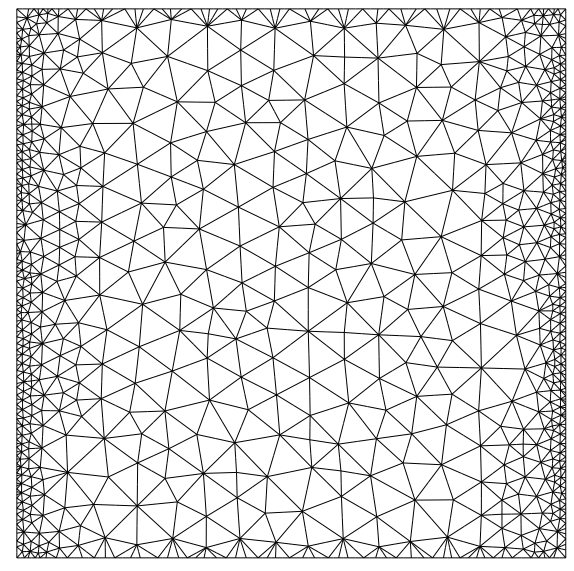

(a) Mesh

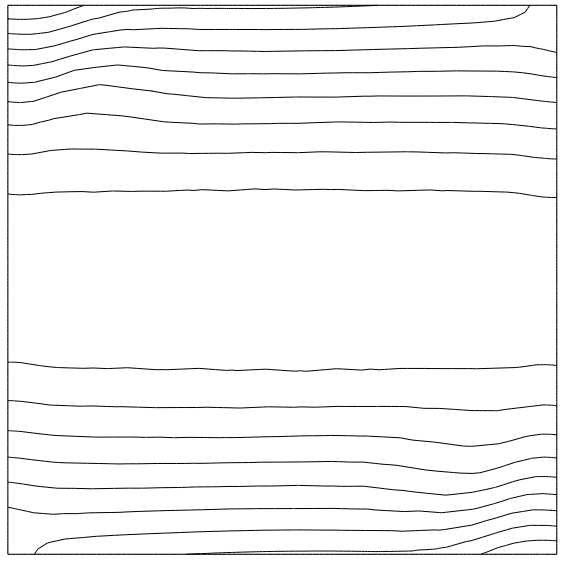

(c) Pressure

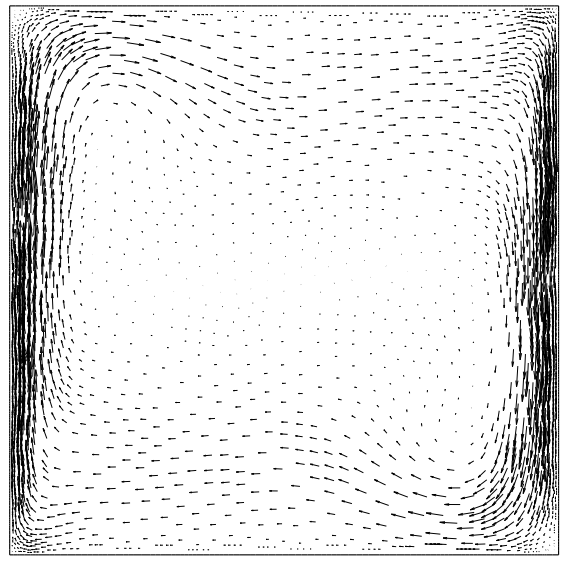

(b) Velocity Vectors

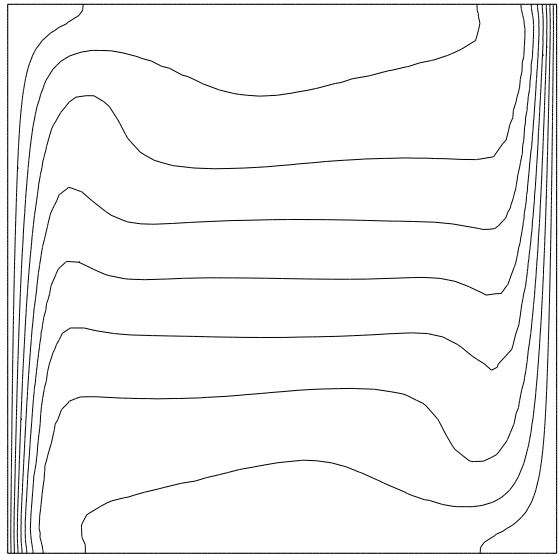

(d) Temperature

Figure 7: Above figures show the mesh, velocity vector plot, temperature contours and pressure contours for a Rayleigh number of $10^{6}$

\begin{tabular}{|c|c|c|c|}
\hline & Present work & Haldenwang $[12,13]$ & Le Quere $[5]$ \\
\hline \hline$U_{\max }$ & 283.0689 & 1082 & 321.9 \\
$y$ & 0.9455 & & 0.928 \\
\hline$V_{\max }$ & 2223.4424 & 2192 & 2222 \\
$x$ & 0.0130 & & 0.012 \\
\hline$N u_{0}$ & 29.6256 & 30.26 & 30.225 \\
\hline$N u_{\max }$ & 91.2095 & & 87.2355 \\
$y$ & 0.0067 & & 0.008 \\
\hline$N u_{\min }$ & 2.0440 & & 1.91907 \\
$y$ & 1.0 & & 1.0 \\
\hline
\end{tabular}

Table 5: The benchmark solution for $R a=10^{8}$ 


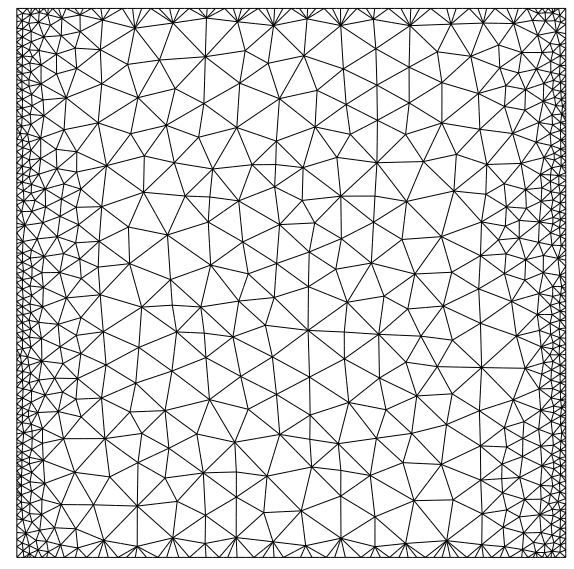

(a) Mesh

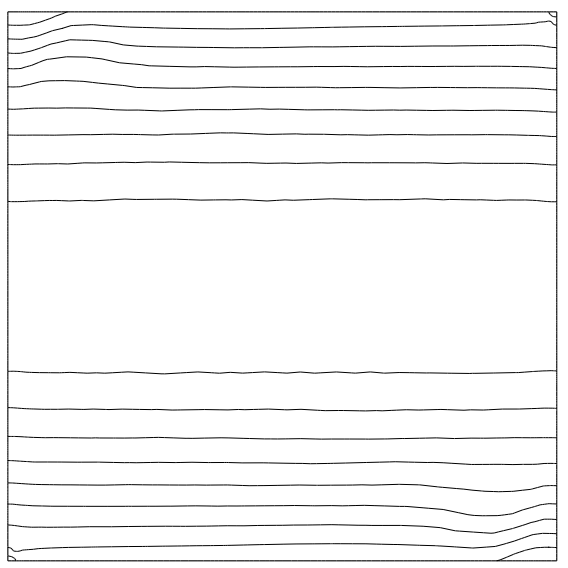

(c) Pressure

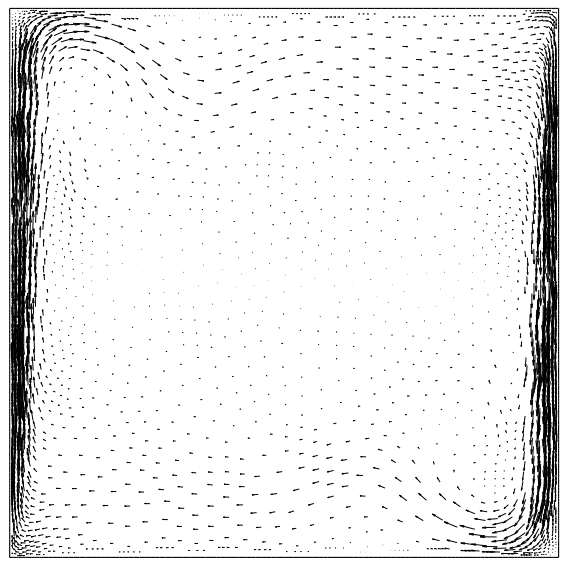

(b) Velocity Vectors

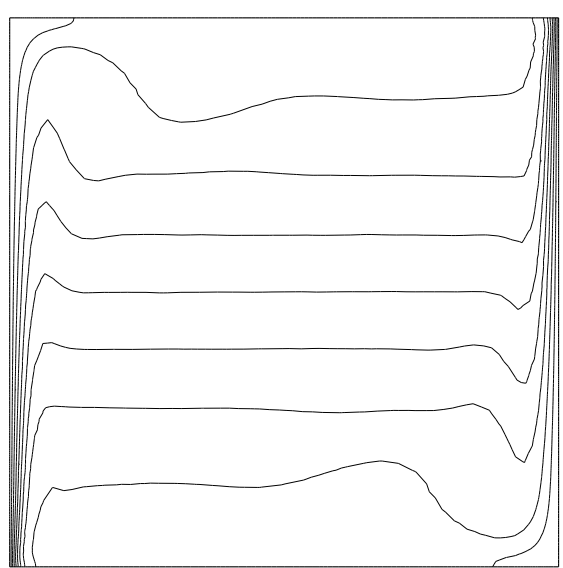

(d) Temperature

Figure 8: Above figures show the mesh, velocity vector plot, temperature contours and pressure contours for a Rayleigh number of $10^{7}$ 


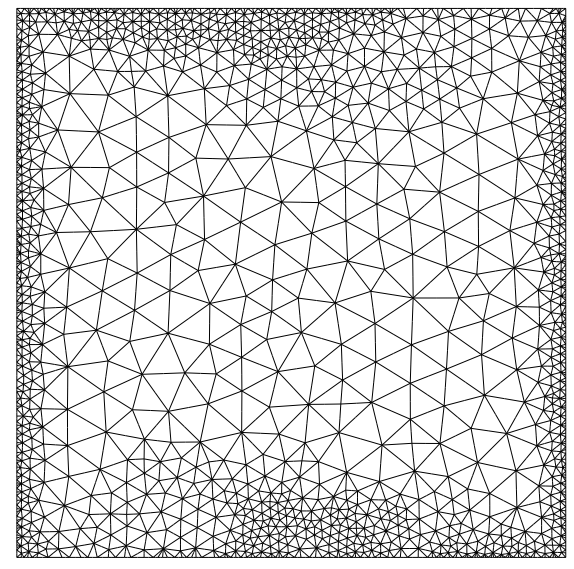

(a) Mesh

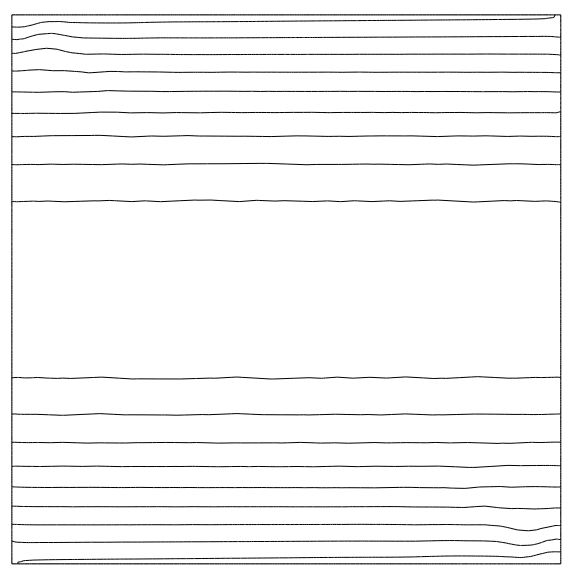

(c) Pressure

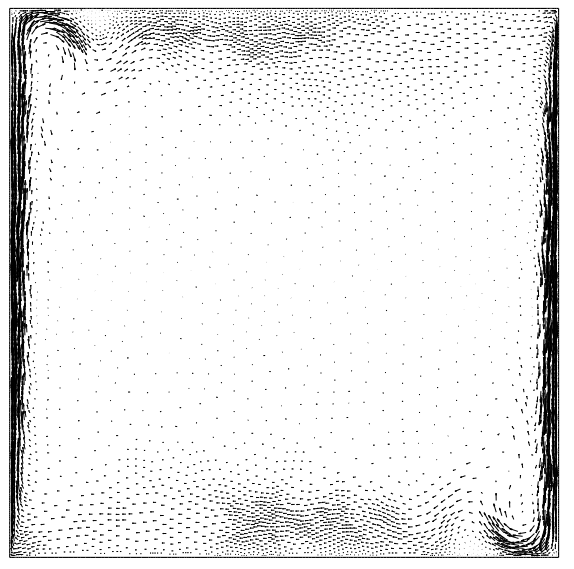

(b) Velocity Vectors

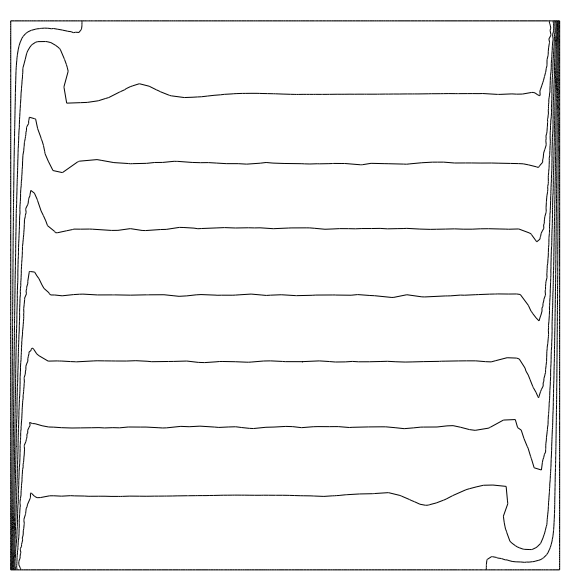

(d) Temperature

Figure 9: Above figures show the mesh, velocity vector plot, temperature contours and pressure contours for a Rayleigh number of $10^{8}$ 


\section{Conclusion}

A full set of results has been produced for the thermally driven cavity problem at Rayleigh numbers of $10^{3}$ to $10^{8}$. The use of h-adaptivity ensures a very accurate solution to this problem at a reasonable computational cost. A pre-adaptive step, adapting the mesh on the basis of the fixed temperature boundary conditions, allows the thin boundary layers to be captured effectively from the very beginning. The results presented compare well with existing transient and steady state solutions both qualitatively and quantitatively. For highly advection dominated problems, such as the high Rayleigh number cases of the thermally driven cavity, h-adaptivity fulfils another very important function, in addition to the vital task of providing optimal meshes. This function is related to the deficiency of standard Galerkin finite element method (analogous to centred difference FDM) in solving flow and transport problems where advection is the dominant mechanism. Many special techniques exist in order to address this deficiency, such as the SUPG method [23] and the Taylor-Galerkin method [24]. However Gresho and Lee [25] have shown that the oscillations that result from using GFEM in advection dominated problems are strongly related to inadequate spatial discretisation. Usmani [26] showed conclusively that for transient solution of advection dominated problems this was indeed the case and the discretisation produced by using $h$-adaptivity made it unnecessary to use any special schemes for advection dominant problems. This finding is confirmed by the results of this paper, where all results have been produced using simply honest GFEM. In the opinion of the authors, this fact makes it doubly attractive to use this method.

\section{References}

[1] G. de Vah1 Davis and I.P.Jones. Natural convection in a square cavity: a comparison exercise. International Journal for Numerical Methods in Fluids, 3:227-248, 1983.

[2] I.P.Jones. A comparison problem for numerical methods in fluid dynamics, the 'double glazing' problem. In First International Conference for Numerical Methods in Thermal Problems, pages 338-348, Swansea,UK, 1979.

[3] T.Saitoh and K.Hirose. High-accuracy bench mark solutions to natural convection in a square cavity. Computational Mechanics, 4:417-427, 1989.

[4] G. de Vahl Davis. Natural convection of air in a square cavity: a benchmark solution. International Journal for Numerical Methods in Fluids, 3:249-264, 1983.

[5] P.Le Quere. Accurate solutions to the square thermally driven cavity at high rayleigh number. Computers and Fluids, 20:29-41, 1991.

[6] D.R.Chenoweth and S.Paolucci. Natural convection in an enclosed vertical air layer with large horizontal temperature differences. Journal of Fluid Mechanics, 169:173$210,1986$. 
[7] S.Paolucci and D.R.Chenoweth. Transition to chaos in a differentially heated vertical cavity. Journal of Fluid Mechanics, 201:379-410, 1989.

[8] P.M.Gresho, R.L.Lee, S.T.Chan, and R.L.Sani. Solution of the time-dependent incompressible navier-stokes and boussinesq equations using the galerkin finite element method. In Proceedings of the IUTAM Symposium on Approximate Methods for Navier-Stokes Problems, pages 203-222, Paderborn,West Germany, September 1979.

[9] R.S.Marshall, J.C.Heinrich, and O.C.Zienkiewicz. Natural convection in a square enclosure by a finite-element penalty function method using primitive fluid variables. Numerical Heat Transfer, 1:315-330, 1978.

[10] A.S.Usmani. Finite Element Modelling of Convective-Diffusive Heat Transfer and Phase Transformation with Reference to Casting Simulation. PhD thesis, University College of Swansea, June 1991.

[11] J.Patterson and J.Imberger. Unsteady natural convection in a rectangular cavity. Journal of Fluid Mechanics, 100:65-86, 1980.

[12] P.Haldenwang. Unsteady numerical simulation by chebyshev spectral methods of natural convection at high rayleigh number. American Society of Mechanical Engineering, Heat Transfer Division, 60:45-51, 1986.

[13] P.Haldenwang and G.Labrosse. 2-d and 3-d spectral chebyshev solutions for free convection at high rayleigh number. In Finite Element Methods in Flow Problems, pages 261-266, 1986.

[14] P.Le Quere and A. De Roquefort. Computation of natural convection in twodimensional cavities with chebyshev polynomials. Journal of Computational Physics, $57: 210-228,1985$.

[15] P.M.Gresho, R.L.Lee, and R.L.Sani. On the time-dependent solution of the incompressible Navier-Stokes equations in two and three dimensions. In Recent Advances in Numerical Methods in Fluids, volume 1. Pineridge Press Limited, Swansea, 1980.

[16] A.S.Usmani, R.W.Lewis, and K.N.Seetharamu. Finite element modelling of naturalconvection-controlled change of phase. International Journal for Numerical Methods in Fluids, 14:1019-1036, 1992.

[17] T.J.R.Hughes. Analysis of transient algorithms with particular reference to stability behaviour. In Computational Methods for Transient Analysis. Elsevier Science Publishers, 1983.

[18] T.J.R.Hughes. The Finite Element Method - Linear Static and Dynamic Finite Element Analysis. Prentice-Hall International, Inc., New Jersey, 1987.

[19] H.C.Huang and A.S.Usmani. The Finite Element Analysis for Heat Transfer. Springer-Verlag, 1994. 
[20] O.C.Zienkiewicz and J.Z.Zhu. Adaptivity and mesh generation. International Journal for Numerical Methods in Engineering, 32:783-810, 1991.

[21] E.Hinton and J.S.Campbell. Local and global smoothing of discontinuous finite element functions using a least squares method. International Journal for Numerical Methods in Engineering, 8:461-480, 1974.

[22] R.W.Lewis, H.C.Huang, A.S.Usmani, and J.T.Cross. Finite element analysis of heat transfer and flow problems using adaptive remeshing including application to solidification problems. International Journal for Numerical Methods in Engineering, $32: 767-781,1991$.

[23] A.N.Brooks and T.J.R.Hughes. Streamline upwind/petrov-galerkin formulations for convection dominated flows with particular emphasis on the incompressible navierstokes equations. Computer Methods in Applied Mechanics and Engineering, 32:199$259,1982$.

[24] J.Donea. A taylor-galerkin method for convective transport problems. International Journal for Numerical Methods in Engineering, 20:101-119, 1984.

[25] P.M. Gresho and R.L.Lee. Don't suppress the wiggles - they are telling you something! Computers and Fluids, 9:223-253, 1981.

[26] A.S.Usmani. Solution of steady and transient advection problems using an h-adaptive finite element method. International Journal of Computational Fluid Dynamics, 11:249-259, 1999 . 